The relationship between nationality and performance in successful attempts to swim across the 'english channel' - a retrospective data analysis from 1875 to 2012

Rüst, Christoph Alexander ; Knechtle, Beat ; Rosemann, Thomas

DOI: https://doi.org/10.5604/17342260.1068224

Posted at the Zurich Open Repository and Archive, University of Zurich ZORA URL: https://doi.org/10.5167/uzh-105890

Journal Article

Published Version

Originally published at:

Rüst, Christoph Alexander; Knechtle, Beat; Rosemann, Thomas (2013). The relationship between nationality and performance in successful attempts to swim across the 'english channel' - a retrospective data analysis from 1875 to 2012. Medicina Sportiva, 17(3):125-133.

DOI: https://doi.org/10.5604/17342260.1068224 


\title{
THE RELATIONSHIP BETWEEN NATIONALITY AND PERFORMANCE IN SUCCESSFUL ATTEMPTS TO SWIM ACROSS THE 'ENGLISH CHANNEL' - A RETROSPECTIVE DATA ANALYSIS FROM 1875 TO 2012
}

\author{
Christoph Alexander Rüst ${ }^{1(\mathrm{C}, \mathrm{D}, \mathrm{E}, \mathrm{F})}$, Beat Knechtle ${ }^{1,2(\mathrm{~A}, \mathrm{~B}, \mathrm{D}, \mathrm{E}, \mathrm{F})}$, Thomas Rosemann $^{1(\mathrm{D}, \mathrm{E})}$ \\ ${ }^{1}$ Institute of General Practice and Health Services Research, University of Zurich, Zurich, Switzerland \\ ${ }^{2}$ Gesundheitszentrum St. Gallen, St. Gallen, Switzerland
}

\begin{abstract}
Objective: To investigate participation and performance trends regarding the nationality of successful solo swimmers (513 women and 1,167 men) in the 'English Channel Swim'.

Methods: The nationality and the total swimming times were analyzed for all swimmers who successfully crossed the English Channel from 1875 to 2012.

Results: The number of successful swimmers increased exponentially across years in both women and men. The highest number of successful female and male swimmers lived in Great Britain, followed by athletes living in the USA and in Australia. The annual fastest women and men improved their swimming times across years. The sex difference in performance remained unchanged at $15 \pm 12 \%$. Between 2001 and 2012, the annual fastest three women and men were not able to improve their swimming times. Men achieved $541 \pm 57 \mathrm{~min}$ and were $55 \pm 47 \mathrm{~min}(13 \pm 7 \%)$ faster than women with $596 \pm 37$ min. Regarding the nationality of the successful swimmers, female swimmers from Great Britain achieved most of the annual fastest swimming times ahead of athletes from the USA and Australia. In men, British swimmers achieved most of the annual fastest swimming times ahead of swimmers from Egypt and the USA. Considering the ten fastest swimmers per country, female swimmers from Australia were the fastest ahead of athletes from USA and Great Britain. In men, the ten fastest swimming times were achieved by swimmers living in the USA, Great Britain and Australia.

Conclusions: Women and men from Great Britain, Australia and the United States of America represented the largest number of successful solo swimmers in the 'English Channel Swims'. Additionally, swimmers from these countries achieved the fastest swimming times.
\end{abstract}

Key words: swimmer, ultra endurance, nationality

\section{Introduction}

With growing interest in ultra-endurance races worldwide, where an ultra-endurance race is defined as an endurance performance exceeding six hours [1], there was also an increasing economical and medical interest in these events [2-4]. Extensive research has already been conducted on the different variables with potential influence on performance in ultra-endurance races such as anthropometric characteristics $[5,6]$, training $[7,8]$ or previous experience [9]. Furthermore, previous research also focused on the effects of age [10$12]$ and sex $[9,13-18]$ on ultra-endurance performance.

It has been shown that origin, nationality or affiliation to a certain ethnic group could also influence the performance in endurance athletes such as longdistance runners [19-21]. An example of the presence of a genetic predisposition determining a superior performance is assumed and partly shown for EastAfrican endurance athletes, who have been dominating long-distance running for more than five decades [22-25]. In running, Kenyan runners dominated running distances from $800 \mathrm{~m}$ to the marathon [23]. The top Kenyan runners seemed to arise from a specific region or ethnic group in Kenya. Most national and international athletes originated from the Rift Valley province and belonged to the Kalenjin ethnic group and Nandi sub-tribe [23]. Apart from running, nationality might play a role also in other sports disciplines such as swimming, where US-American and Australian swimmers dominated the 2000 Olympic Games [26,27].

In swimming, apart from events held in pools, open-water ultra-swimming has a long tradition such as swimming the English Channel [28-30]. Since 1875, athletes cross the 'English Channel'. To date, no study investigated the participation and performance trends in open-water ultra-endurance swimmers participating in the 'English Channel Swim' regarding the aspect of the nationality of the swimmers. Considering the relationship between nationality and performance in ultra-endurance races, recent studies investigated ultra-marathoners [31-34] and ultra-endurance 
athletes competing in multi-sports disciplines such as duathlon [35] and triathlon [36-41]. A common finding in these studies on ultra-endurance athletes was that European athletes were dominating both participation and performance [32,37-41]. Another important finding in these studies was that competitors were mainly originating from the country where the ultra-endurance race was held [31-36] or from neighboring countries [32,34-36].

We therefore investigated participation and performance trends for successful solo swimmers in the 'English Channel Swim' regarding the nationality of the swimmers. The aims of the present study were, firstly, to investigate the nationality of participants in the 'English Channel Swim' from 1875-2012 and, secondly, to analyze the performance and performance trends of the swimmers of the leading countries. We firstly hypothesized that British and French swimmers would mainly compete in the 'English Channel Swim' because they live next to the English Channel'. A second hypothesis was that swimmers from leading swim nations such as America and Australia would dominate the 'English Channel Swim'.

\section{Methods}

The nationality and the swimming times were analyzed for all participants in 'English Channel Swim' from 1875 to 2012. The data set from this study was obtained from the website of the Channel Swimming Association [28]. The study was approved by the institutional review board of St. Gallen, Switzerland, with waiver of the requirement for informed consent given that the study involved the analysis of publicly available data.

\section{The 'English Channel Swim'}

The usual route of the 'English Channel Swim', from Dover (Great Britain) to Calais (France), starts at Shakespeare Beach (Great Britain), one hour before or one hour after high water and ends in Cap Gris Nez (France). The shortest distance to cross the 'English Channel' is 18.2 nautical miles, equal to $33.8 \mathrm{~km}$. In the course of a year, the water temperature of the Channel varies between $15^{\circ} \mathrm{C}$ and $18^{\circ} \mathrm{C}$ during summer [42]. Therefore, athletes are recommended to acclimate to this temperature during their trainings. If a swimmer wants to participate in the 'English Channel Swim', she/he first needs to fill in an enquiry form, in which she/he has to demonstrate his/her efforts of training to make a realistic attempt to meet the requirements to cross the 'English Channel'. A medical certificate has to be added to affirm the swimmers physical integrity. Athletes corresponding to these terms and conditions are summoned up to an observed one hour swim and evaluated by a committee whether they can attend to the 'English Channel Swim' or not. Furthermore, athletes have to agree to the rules of the association and they also have to be escorted by a vessel, whose pilot is registered to the association.

\section{Data analysis}

All solo swimmers who ever participated in the 'English Channel Swim' between 1875 and 2012 were regarded. From all countries with more than ten men or women the performances of the overall top ten athletes were compared. These countries were Great Britain (GBR), United States of America (USA), Australia (AUS), Canada (CAN), Ireland (IRL), India (IND), Mexico (MEX), Germany (GER), Japan (JPN) and The Netherlands (NED) for women, and Great Britain (GBR), United States of America (USA), Australia (AUS), Ireland (IRL), Republic of South Africa (RSA), Egypt (EGY), India (IND), France (FRA), Mexico (MEX), Brasilia (BRA), The Netherlands (NED), Belgium (BEL), Japan (JPN), Czech Republic (CZE), Argentina (ARG), Canada (CAN), Spain (ESP) and New Zealand (NZL) for men.

The change in swim times across years of the annual top three women and men per country was analyzed between 2001 and 2012. Only Great Britain (GBR) and the United States of America (USA) could provide enough successful swimmers for this analysis. To compare these results with the overall population, the number of athletes and the performance of the annual fastest women and men were analyzed. The performance of the annual top three women and men was analyzed between 2001 and 2012. During the 127 years period between 1875 and 2012, only in 78 years 'English Channel Swims' were held. The change in the number of swimmers was analyzed regarding the number of years where 'English Channel Swims' were held. The year 1875 was defined as event year ' 0 ' and 2012 was defined as event year ' 78 '. In contrast, the change in performance was analyzed regarding the absolute year (1875='0'; 2012='127').

The sex difference in performance was calculated using the equation ([swimming time ( $\mathrm{min}$ ) in women] - [swimming time (min) in men]) / [swimming time $(\min )$ in $\mathrm{men}] \times 100$, where the sex difference was calculated for every pairing of equally placed athletes (e.g. between annual fastest male and female swimmer, between annual second fastest male and female, etc.) before calculating mean value and standard deviation of all the pairings. In order to facilitate reading all sex differences were transformed to absolute values before analysing.

\section{Statistical analysis}

In order to increase the reliability of the data analyses, each set of data was tested for normal distribution and for homogeneity of variances prior to statistical analyses. Normal distribution was tested using 
a D'Agostino and Pearson omnibus normality test and homogeneity of variances was tested using a Levene's test in case of two groups and using a Bartlett's test in case of more than two groups. Data in the text are given as mean \pm standard deviation (SD). To test significant changes in a variable across years, single linear regression analysis was used. To find differences between multiple groups, a one-way analysis of variance (ANOVA) with subsequent Tukey-Kramer post-hoc analysis was used. Statistical analyses were performed using IBM SPSS Statistics (Version 19, IBM
SPSS, Chicago, IL, USA) and GraphPad Prism (Version 5, GraphPad Software, La Jolla, CA, USA). Significance was accepted at $P<0.05$ (two-sided for $t$-tests).

\section{Results}

In total, data were available for 1,680 athletes. From the 513 women and 1,167 men, swim times and nationality were available. Between 1875 and 2012, the number of successful swimmers increased exponentially in both women and men (Fig. 1). For both women (Fig. 2A) and men (Fig. 2B) most of the

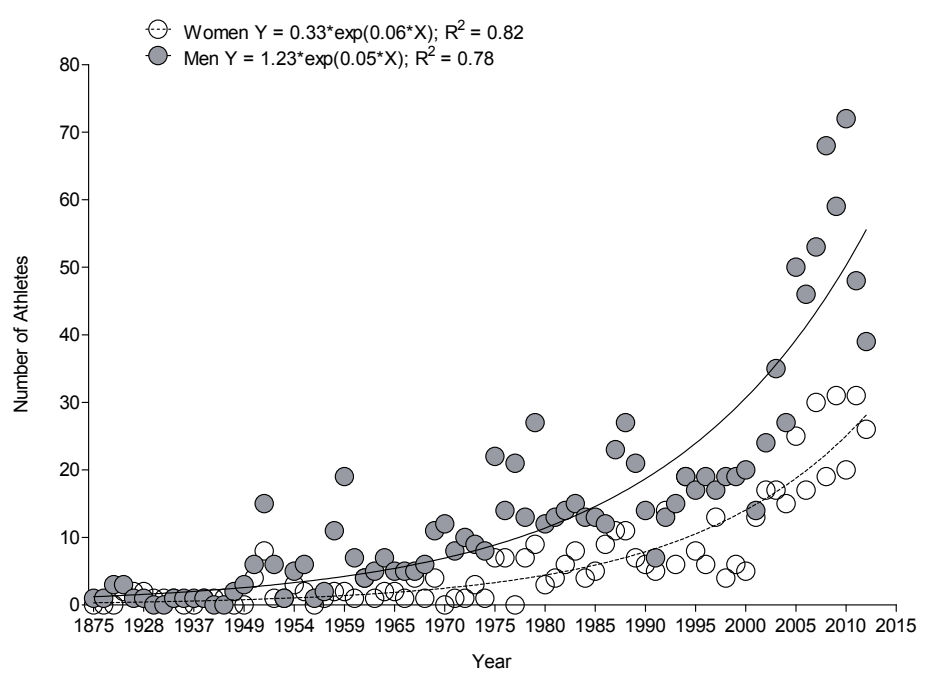

Fig. 1. Number of annual women and men participating in the 'English Channel Swim'

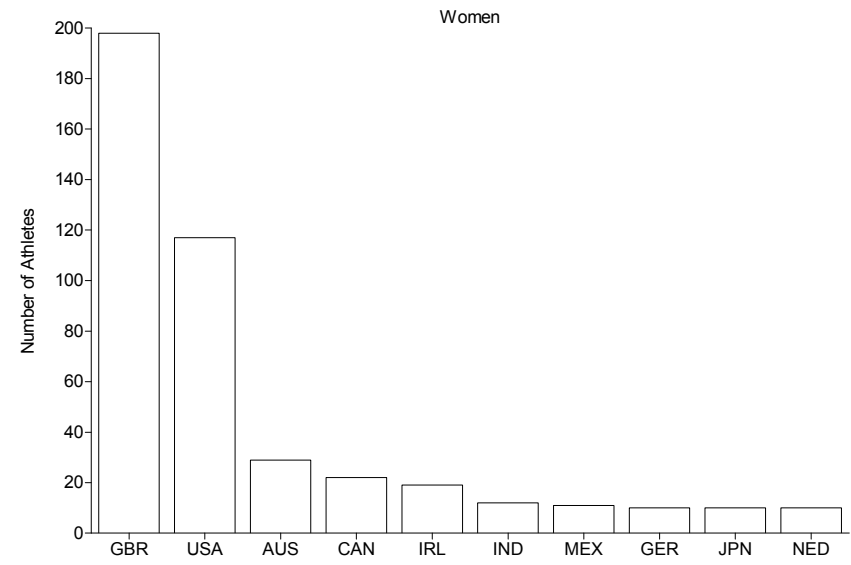

A

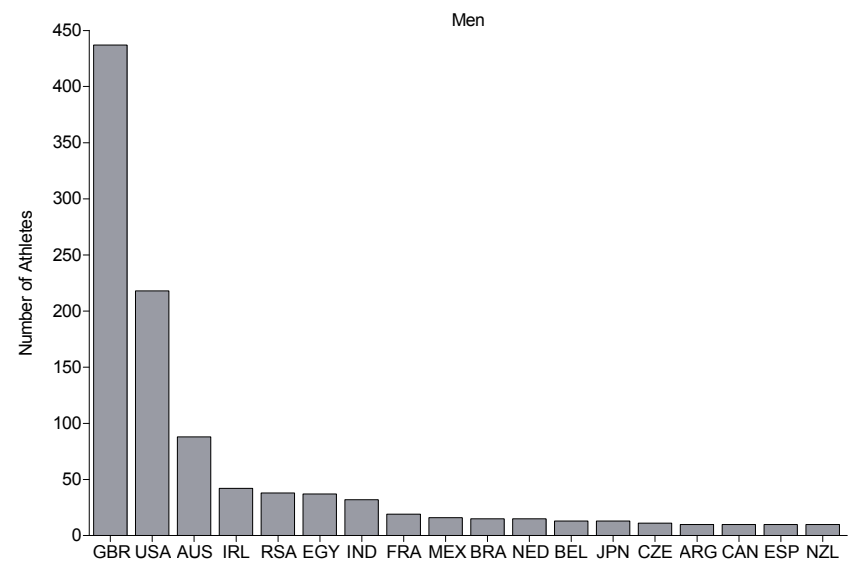

B 


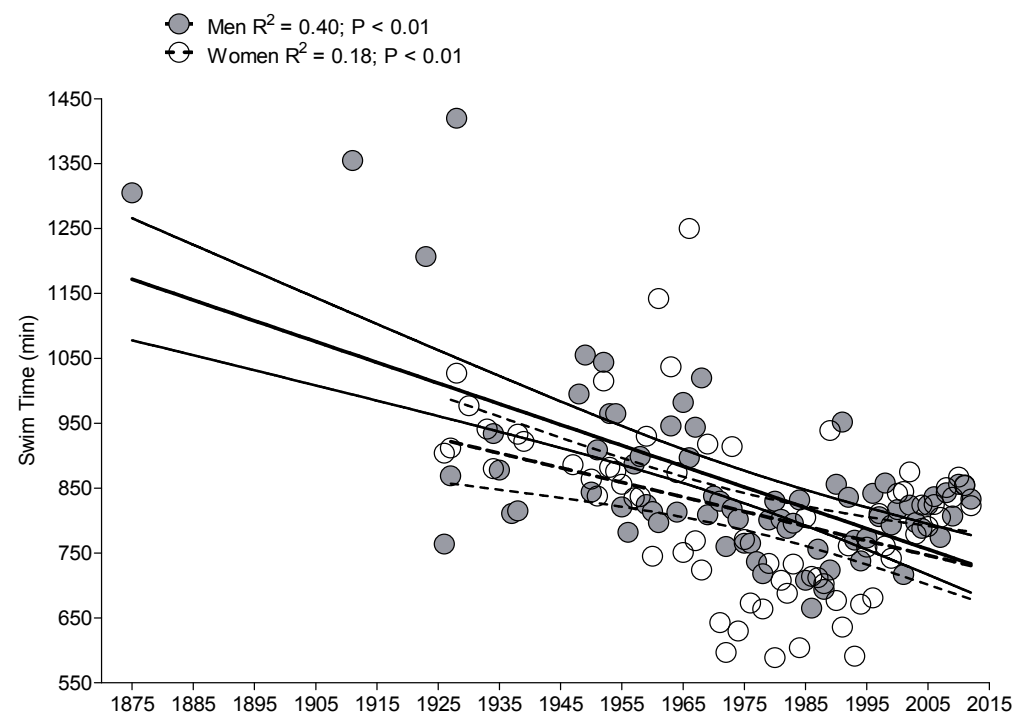

Fig. 3. Annual overall performances with linear regression and $95 \% C I$

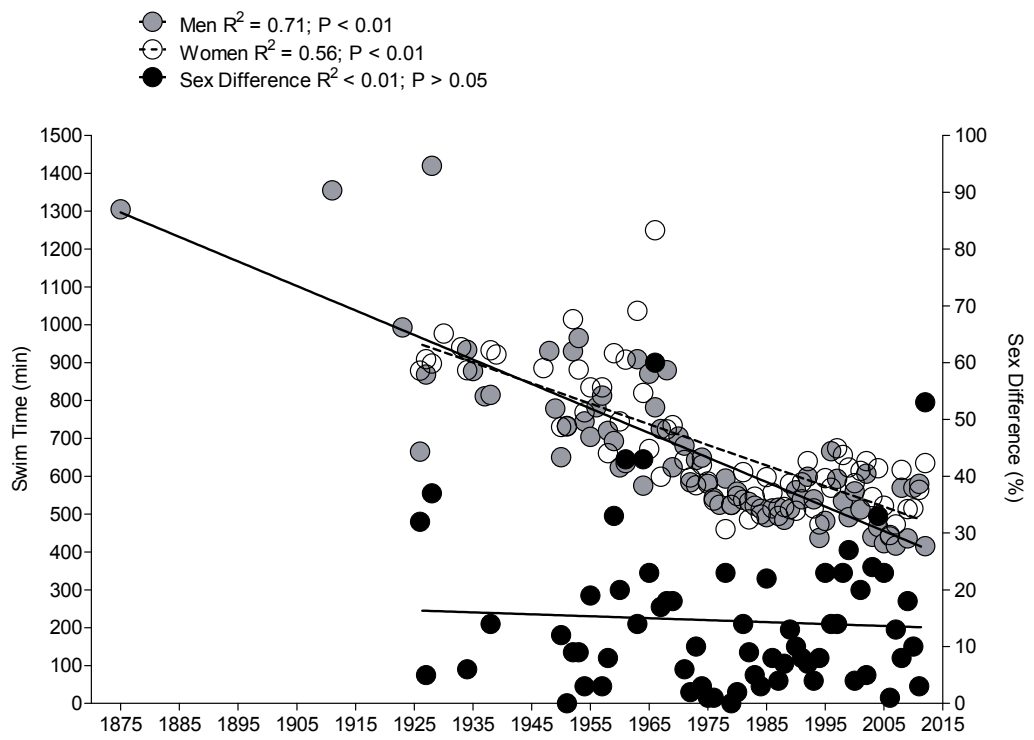

Year

Fig. 4. Performance of the annual fastest women and men with sex difference

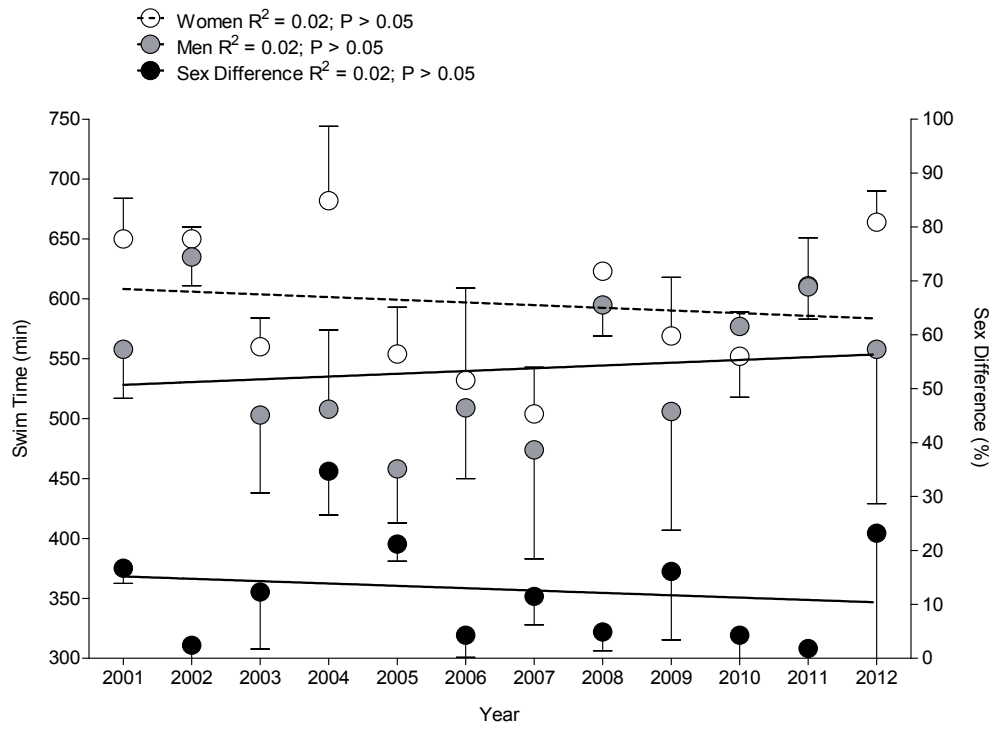

Fig. 5. Performance of the annual top three women and men between 2001 and 2012 with sex difference 


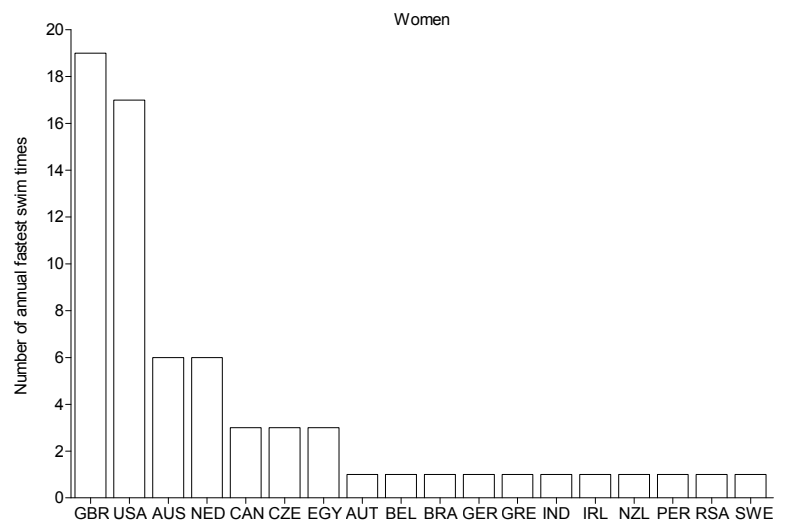

A

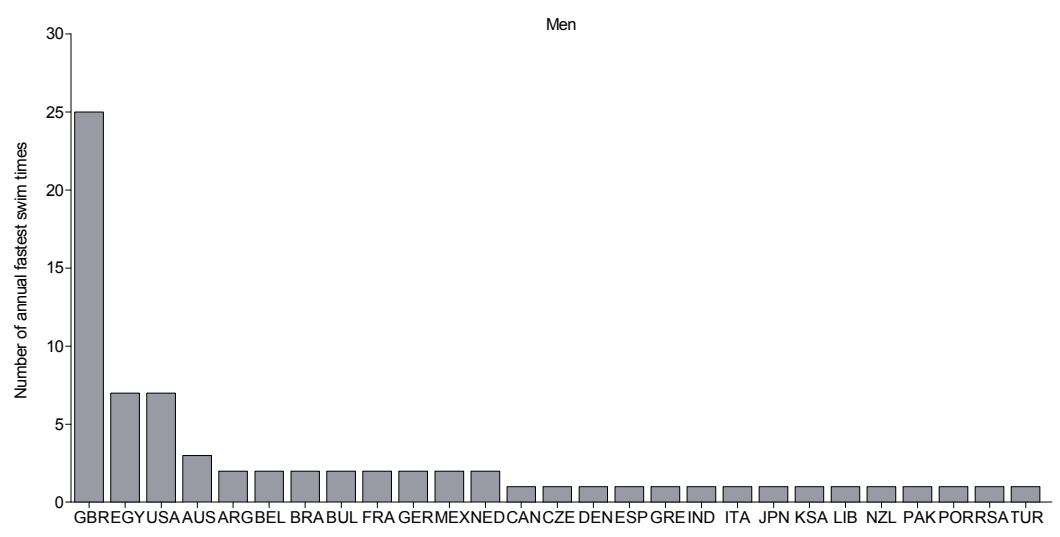

B

Fig. 6. Number of annual fastest swim times by nationality for women (Panel A) and men (Panel B)

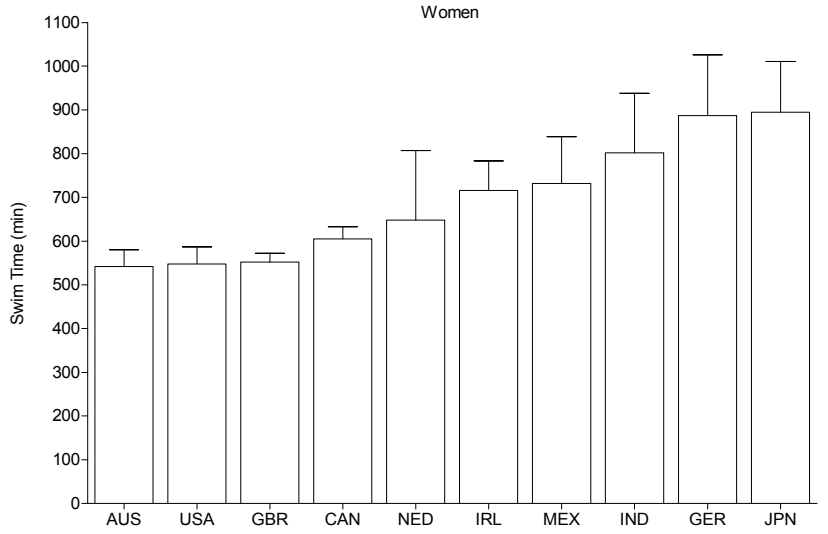

A

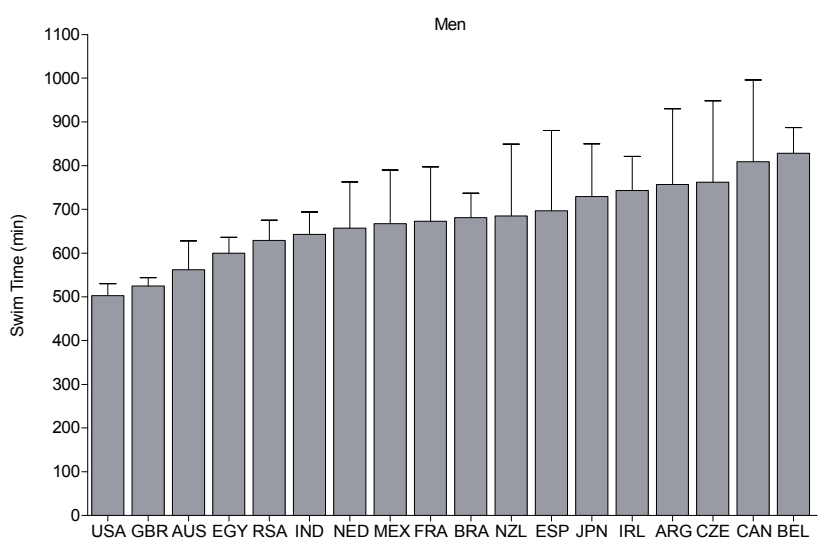

B

Fig. 7. Performance of the overall top ten women (Panel A) and men (Panel B) per country. All countries with more than ten women or men athletes are indicated 


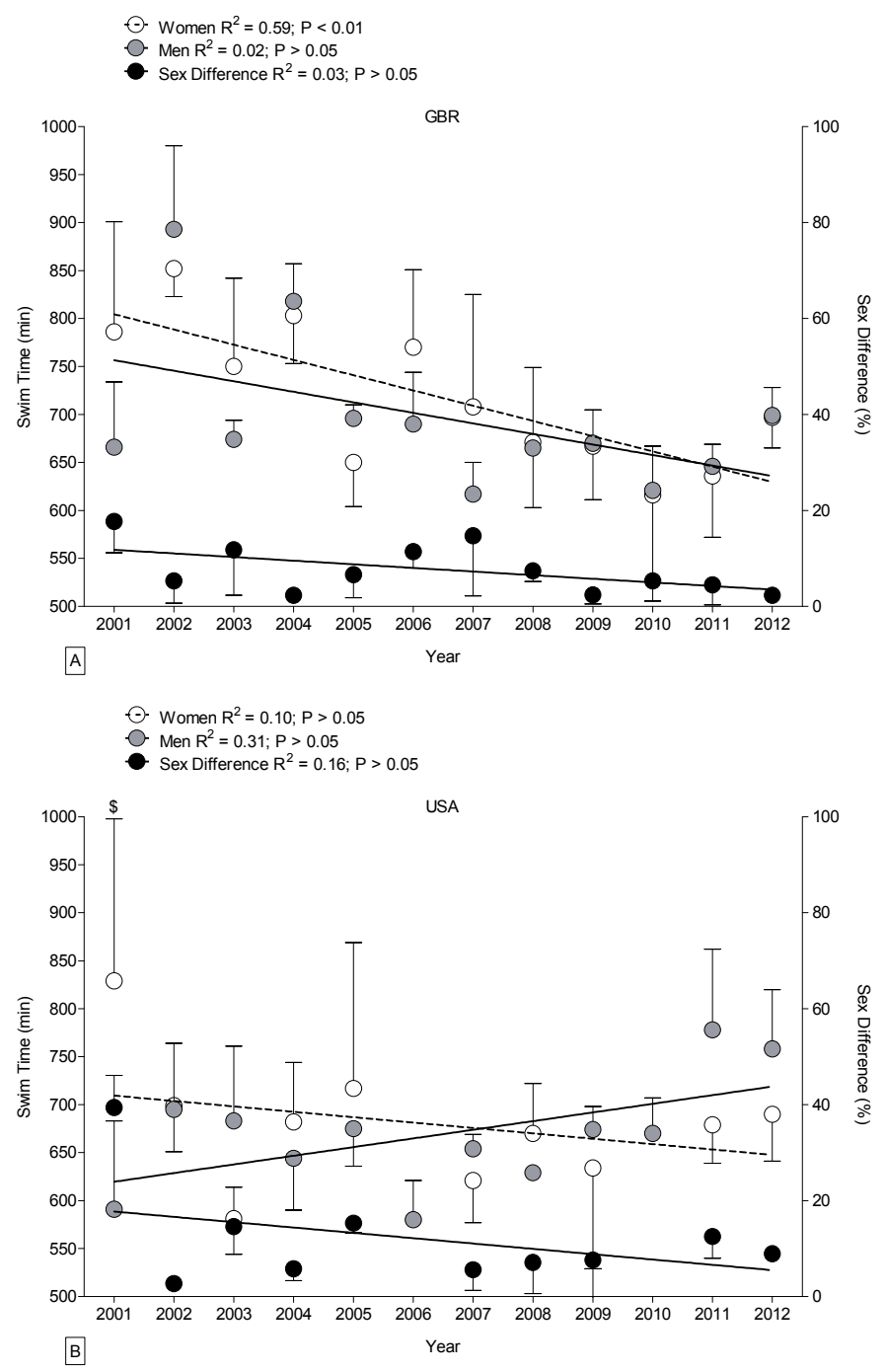

Fig. 8. Performance of the annual top three men and women with sex difference for the two countries with at least three athletes per year between 2001 and 2012 (GBR and USA). In 2006 and 2010 less than three women originated from the USA

successful swimmers lived in Great Britain, followed by athletes living in the USA and in Australia.

Over time, both the annual overall women and men (Fig. 3) and the annual fastest women and men (Fig. 4) improved their swim times. Although both the annual fastest women and men improved their swimming times, the sex difference in performance remained unchanged at $15 \pm 12 \%$ (Fig. 4). Between 2001 and 2012, the annual fastest three women and men were not able to improve their swimming times (Fig. 5). Men achieved $541 \pm 57 \mathrm{~min}$ and women $596 \pm 37 \mathrm{~min}$. Men were $55 \pm 47 \min (13 \pm 7 \%)$ faster than women.

Considering the nationality of the swimmers, female athletes from Great Britain achieved the highest number of the annual fastest swimming times ahead of athletes living in the USA and Australia (Fig. 6A). In men, British swimmers achieved most of the annual fastest swimming times ahead of swimmers living in Egypt and the USA (Fig. 6B). Considering the ten fastest swimmers per country, female swimmers living in Australia were the fastest ahead of swimmers living in USA and Great Britain (Fig. 7A). In men, the ten fastest swimming times were achieved by swimmers living in the USA, Great Britain and Australia (Fig. 7B).

Figure 8 presents the change in performance for the annual top three British (Panel A) and the annual top three American (Panel B) swimmers. Female swimmers from Great Britain improved swimming times across years, but not male swimmers. The sex difference remained unchanged at $7.6 \pm 4.7 \%$. In USAmerican swimmers, neither women nor men improved swimming times; the sex difference remained unchanged at $12.0 \pm 4.6 \%$.

\section{Discussion}

This study intended to investigate the origin of the fastest swimmers in the 'English Channel Swim'. The main findings were that (i) participation in successful finishers increased exponentially over time for both women and men, (ii) most of the successful finishers lived in Great Britain, (iii) swimming times decreased for both women and men over time, (iv) the fastest 
swimming times were achieved by athletes living in Australia, USA and Great Britain and $(v)$ British women improved swimming times in the last 12 years.

\section{Exponential increase in participation}

The participation increased in an exponential manner in the last 20 years in the 'English Channel Swim' or both women and men. In comparable ultraendurance challenges such as the 'Western States 100Mile Endurance Run' between 1974 and 2007 [43] or the ultra-triathlons worldwide between 1985 and 2009 [16], the participation showed a rather linear increase with a stabilization in the last 20 years.

The 'English Channel Swim' may have developed a special myth in the last years since it is also called the 'Everest' of swimming [28]. Since the first 'English Channel Swim', six fatalities ( $\sim 0.4 \%$ of the starters) occurred until 2011, which is, however, considerably lower compared to the $1.3 \%$ fatalities on the 'Mount Everest' [44]. Regarding the participation in women and men, men's participation increased in a more pronounced exponential manner in the last ten years. Most probably, the motivation to compete in ultra-endurance races differs between women and men [45].

Regarding the participation by nationality, a first important finding was that British swimmers represented the highest number of successful solo swimmers for both women and men. In addition, female and male British swimmers improved their swimming times across years. In contrast to our hypothesis, French swimmers were not among the leading nations regarding both participation and performance. Swimmers can cross the 'Channel' from England to France or from France to England [28]. Most swimmers crossed the 'Channel' from England to France [46]. Due to the heavy traffic in the 'Channel' where more than 500 vessels pass through the shipping lanes each day [47], France banned swimmers using the Calais to Dover route 17 years ago [48]. This might be the main reason that French swimmers were only on $12^{\text {th }}$ position regarding the number of successful solo swimmers.

The most probable explanation for the high frequency of British swimmers to participate in the 'English Channel Swim' is the vicinity of the Channel for British swimmers. British athletes can reach Dover by car, train or plane. The Port of Dover is located $\sim 128$ kilometers from London's Heathrow Airport ( $\sim 2$ to 2 1/2 hours). Regarding American athletes, they have to arrive by plane. Most probably, Americans can rather afford the expenses of flight, accommodation and support for the 'English Channel Swim' compared to swimmers from other countries since the average salary of Americans is one of the highest in the World compared to participants from others countries [49].

\section{The fastest swimmers originate from Australia, USA and Great Britain}

A second hypothesis was that swimmers from leading swim nations such as America and Australia would dominate the 'English Channel Swim' since swimmers from America and Australia are dominating indoor pool swimming [26,27]. The fastest swimming times in the 'English Channel Swim' were achieved by athletes living in Australia, the United States of America and Great Britain. Obviously, Australian and American swimmers are also able to achieve fast swimming times in open-water ultradistance swimming.

An interesting finding was that British women were able to improve their swimming times between 2001 and 2012 whereas British men and both American women and men could not improve in the last twelve years. This finding might be explained by social or motivational aspects needing further investigations. A part of the reason for this may be the tides. On the whole the fastest swims are completed in around $12 \mathrm{~h}$ (two tides) then it's either 18 or $24 \mathrm{~h}$ (3-4 tides).

Although both the annual overall women and men and the annual fastest women and men improved their swimming times, the annual three fastest women and men were not able to improve in the last 12 years. The sex difference for the annual top three women and men overall was $13 \pm 7 \%$, slightly higher than the sex difference for the annual top three British swimmers $(7.6 \pm 4.7 \%)$ and the annual top three American swimmers $(12.0 \pm 4.6 \%)$.

\section{Limitations}

This cross-sectional study is limited since variables such as age $[11,12,17]$, anthropometric characteristics $[6-9,14]$, training $[6-9,14,15]$, and previous experience [7-9] with an effect on ultra-endurance performance were not included. In addition, changes in environmental conditions $[50,51]$ across the years might have had an influence on swim times. The motivation of these athletes is also not known [45].

\section{Conclusions}

To summarize, both female and male swimmers from Great Britain and the United States of America represented the largest number of successful solo swimmers in the 'English Channel Swim' since 1875. The fastest swimming times in 'English Channel Swim' were achieved by swimmers living in Australia, the United States of America and Great Britain. Future studies need to investigate what motivates these swimmers to cross the 'English Channel'.

\section{Author disclosures}

The authors have no conflict of interest and received no external funding. 


\section{References}

1. Zaryski C, Smith DJ. Training principles and issues for ultra-endurance athletes. Curr Sports Med Rep 2005; 4: 165-70.

2. Burns J, Keenan AM, Redmond AC. Factors associated with triathlon-related overuse injuries. J Orthop Sports Phys Ther 2003; 33: 177-84.

3. Egermann M, Brocai D, Lill CA, et al. Analysis of injuries in long-distance triathletes. Int J Sports Med 2003; 24: 271-6.

4. Strock GA, Cottrell ER, Lohman JM. Triathlon. Phys Med Rehabil Clin N Am 2006; 17: 553-64.

5. Knechtle B, Knechtle P, Andonie JL, et al. Influence of anthropometry on race performance in extreme endurance triathletes: World Challenge Deca Iron Triathlon 2006. Br J Sports Med 2007; 41: 644-8.

6. Knechtle B, Knechtle P, Rosemann T. Upper body skinfold thickness is related to race performance in male Ironman triathletes. Int J Sports Med 2011; 32: 20-7.

7. Knechtle B, Wirth A, Baumann B, et al. Differential correlations between anthropometry, training volume, and performance in male and female Ironman triathletes. J Strength Cond Res 2010; 24: 2785-93.

8. Knechtle B, Knechtle P, Rosemann T, et al. Personal best time, not anthropometry or training volume, is associated with total race time in a triple iron triathlon. J Strength Cond Res 2011; 25: 1142-50.

9. Knechtle B, Wirth A, Baumann B, et al. Personal best time, percent body fat, and training are differently associated with race time for male and female ironman triathletes. Res Q Exerc Sport 2010; 81: 62-8.

10. Haupt S, Knechtle B, Knechtle P, et al. The age-related performance decline in ultra-endurance mountain biking. Res Sports Med 2013; 21: 146-58.

11. Knechtle B, Rüst CA, Rosemann T, et al. Age-related changes in 100-km ultra-marathon running performance. Age (Dordr) 2012; 34: 1033-45.

12. Lepers R, Rüst CA, Stapley PJ, et al. Relative improvements in endurance performance with age: evidence from 25 years of Hawaii Ironman racing. Age (Dordr) 2013; 35: 953-62.

13. Eichenberger E, Knechtle B, Knechtle P, et al. No gender difference in peak performance in ultra-endurance swimming performance - Analysis of the 'Zurich 12-h Swim' from 1996 to 2010. Chin J Physiol 2012; 55: 346-51.

14. Knechtle B, Baumann B, Knechtle P, et al. Speed during training and anthropometric measures in relation to race performance by male and female open-water ultra-endurance swimmers. Percept Mot Skills 2010; 111: 463-74.

15. Knechtle B, Baumann B, Knechtle P, et al. What influences race performance in male open-water ultra-endurance swimmers: anthropometry or training? Human Movement 2010; 11: 91-5.

16. Knechtle B, Knechtle P, Lepers R. Participation and performance trends in ultra-triathlons from 1985 to 2009. Scand J Med Sci Sports 2011; 21: e82-e90.

17. Lepers R, Maffiuletti NA. Age and gender interactions in ultraendurance performance: insight from the triathlon. Med Sci Sports Exerc 2011; 43: 134-9.

18. Rüst CA, Knechtle B, Knechtle P, et al. Gender difference and age-related changes in performance at the long distance duathlon World Championships. J Strength Cond Res 2013; 27: 293-301.

19. Caló MC, Vona G. Gene polymorphisms and elite athletic performance. J Anthropol Sci 2008; 86: 113-31.

20. Eynon N, Ruiz JR, Oliveira J, et al. Genes and elite athletes: a roadmap for future research. J Physiol 2011; 589: 3063-70.

21. Scott RA, Pitsiladis YP. Genotypes and distance running : clues from Africa. Sports Med 2007; 37: 424-7.

22. Larsen HB. Kenyan dominance in distance running. Comp Biochem Physiol A Mol Integr Physiol 2003; 136: 161-70.

23. Onywera VO, Scott RA, Boit MK, et al. Demographic characteristics of elite Kenyan endurance runners. J Sports Sci 2006; 24: 415-22.
24. Scott RA, Fuku N, Onywera VO, et al. Mitochondrial haplogroups associated with elite Kenyan athlete status. Med Sci Sports Exerc 2009; 41: 123-8.

25. Scott RA, Georgiades E, Wilson RH, et al. Demographic characteristics of elite Ethiopian endurance runners. Med Sci Sports Exerc 2003; 35: 1727-32.

26. Trewin CB, Hopkins WG, Pyne DB. Relationship between world-ranking and Olympic performance of swimmers. $J$ Sports Sci 2004; 22: 339-45.

27. Pyne D, Trewin C, Hopkins W. Progression and variability of competitive performance of Olympic swimmers. J Sports Sci 2004; 22: 613-20.

28. Channel Swimming, www.dover.uk.com/channelswimming

29. Eichenberger E, Knechtle B, Knechtle P, et al. Best performances by men and women open-water swimmers during the 'English Channel Swim' from 1900 to 2010. J Sports Sci 2012; 30: 1295-301.

30. Fischer G, Knechtle B, Rüst CA, et al. Male swimmers cross the English Channel faster than female swimmers. Scand J Med Sci Sports 2013; 23: e48-55.

31. Knoth C, Knechtle B, Rüst CA, et al. Participation and performance trends in multistage ultramarathons - the 'Marathon des Sables' 2003-2012. Extrem Physiol Med 2012; 1: 13.

32. Abou Shoak M, Knechtle B, Rüst CA, et al. European dominance in multistage ultramarathons: an analysis of finisher rate and performance trends from 1992 to 2010. Open Access J Sports Med 2013; 4: 9-18.

33. Knechtle B, Rüst CA, Rosemann T. The aspect of nationality in participation and performance in ultra-marathon running - A comparison between 'Badwater' and 'Spartathlon'. $O A$ Sports Medicine 2013; 1: 1.

34. Eichenberger E, Knechtle B, Rüst CA, et al. The aspect of nationality and performance in a mountain ultra-marathon - the ,Swiss Alpine Marathon. J Hum Sport Exerc 2012; 7: 748-62.

35. Rüst CA, Knechtle B, Knechtle P, et al. The aspect of nationality in participation and performance at the 'Powerman Duathlon World Championship' - The 'Powerman Zofingen' from 2000 to 2011. J Sci Cycling 2012; 2: 33-9.

36. Jürgens D, Knechtle B, Rüst CA, et al. An analysis of participation and performance by nationality at 'Ironman Switzerland' from 1995 to 2011. J Sci Cycling 2012; 1: 10-20.

37. Lenherr R, Knechtle B, Rüst CA, et al. From Double to Double Deca Iron ultra-triathlon - A retrospective data analysis from 1985 to 2011. Physical Culture and Sport. Studies and Research 2012; 54: 55-67.

38. Rüst CA, Knechtle B, Knechtle P, et al. European athletes dominate performance in Double Iron ultra-triathlons A retrospective data analysis from 1985 to 2010. Eur J Sport Sci DOI:10.1080/17461391.2011.641033.

39. Jeffery S, Knechtle B, Rüst CA, et al. European dominance in Triple Iron ultra-triathlons from 1988 to 2011. J Sci Cycling 2012; 1: 30-8.

40. Lepers R, Knechtle B, Knechtle P, et al. Analysis of ultra-triathlon performance. Open Access J Sports Med 2011; 2: 131-6.

41. Sigg K, Knechtle B, Rüst CA, et al. Central European triathletes dominate Double Iron ultratriathlon - analysis of participation and performance 1985-2011. Open Access J Sports Med 2011; 3: 159-68.

42. The Channel Swimming Association, www.channelswimmingassociation.com

43. Hoffman MD, Wegelin JA. The Western States 100-Mile Endurance Run: participation and performance trends. Med Sci Sports Exerc 2009; 41: 2191-8.

44. Firth PG, Zheng H, Windsor JS, et al. Mortality on Mount Everest, 1921-2006: descriptive study. BMJ 2008; 337: a2654.

45. Krouse RZ, Ransdell LB, Lucas SM, et al. Motivation, goal orientation, coaching, and training habits of women ultrarunners. J Strength Cond Res 2011; 25: 2835-42.

46. Channel Swimming, www.dover.uk.com/channelswimming/ swims

47. Channel Traffic, www.dover.uk.com/channeltraffic 
48. Ban on swims across Channel urged by French coastguard, www.bbc. co.uk/news/uk-england-kent-11664240

49. World salaries, www.worldsalaries.org

50. Parise CA, Hoffman MD. Influence of temperature and performance level on pacing a $161 \mathrm{~km}$ trail ultramarathon. Int $J$ Sports Physiol Perform 2011; 6: 243-51.

51. Wegelin JA, Hoffman MD. Variables associated with odds of finishing and finish time in a 161-km ultramarathon. Eur J Appl Physiol 2011; 111: 145-53.

Accepted: September 18, 2013

Published: September 27, 2013
Address for correspondence: PD Dr. med. Beat Knechtle Facharzt FMH für Allgemeinmedizin

Gesundheitszentrum St. Gallen

Vadianstrasse 26

9001 St. Gallen

Switzerland

Telefon $+41(0) 712268282$

Telefax +41 (0) 712268272

e-mail: beat.knechtle@hispeed.ch

Christoph A. Rüst: christoph.ruest@bluewin.ch Beat Knechtle: beat.knechtle@hispeed.ch

Thomas Rosemann: thomas.rosemann@usz.ch 